Article

\title{
New Insights on the Feature and Function of Tail Tubular Protein B and Tail Fiber Protein of the Lytic Bacteriophage $\varphi$ YeO3-12 Specific for Yersinia enterocolitica Serotype 0:3
}

\author{
Anna Pyra ${ }^{1, *}{ }^{\infty}$, Karolina Filik ${ }^{2}{ }^{(}$, , Bożena Szermer-Olearnik ${ }^{2}$, Anna Czarny ${ }^{2}$ and \\ Ewa Brzozowska 2,*(D) \\ 1 Faculty of Chemistry, University of Wroclaw, 14 F. Joliot-Curie St, 50383 Wroclaw, Poland \\ 2 Hirszfeld Institute of Immunology and Experimental Therapy, Polish Academy of Sciences, \\ 12 R. Weigl St, 53114 Wroclaw, Poland; karolina.filik@hirszfeld.pl (K.F.); \\ bozena.szermer-olearnik@hirszfeld.pl (B.S.-O.); anna.czarny@hirszfeld.pl (A.C.) \\ * Correspondence: anna.pyra@chem.uni.wroc.pl (A.P.); ewa.brzozowska@hirszfeld.pl (E.B.); \\ Tel.: +48-71-375-7240 (A.P.); +48-71-337-1172 (E.B.)
}

Received: 29 August 2020; Accepted: 23 September 2020; Published: 24 September 2020

check for updates

\begin{abstract}
For the first time, we are introducing TTPBgp12 and TFPgp17 as new members of the tail tubular proteins B (TTPB) and tail fiber proteins (TFP) family, respectively. These proteins originate from Yersinia enterocolitica phage $\varphi$ YeO3-12. It was originally thought that these were structural proteins. However, our results show that they also inhibit bacterial growth and biofilm formation. According to the bioinformatic analysis, TTPBgp12 is functionally and structurally similar to the TTP of Enterobacteria phage T7 and adopts a $\beta$-structure. TFPgp17 contains an intramolecular chaperone domain at its C-terminal end. The N-terminus of TFPgp17 is similar to other representatives of the TFP family. Interestingly, the predicted 3D structure of TFPgp17 is similar to other bacterial S-layer proteins. Based on the thermal unfolding experiment, TTPBgp12 seems to be a two-domain protein that aggregates in the presence of sugars such as maltose and N-acetylglucosamine (GlcNAc). These sugars cause two unfolding events to transition into one global event. TFPgp17 is a one-domain protein. Maltose and GlcNAc decrease the aggregation temperature of TFPgp17, while the presence of $\mathrm{N}$-acetylgalactosamine (GalNAc) increases the temperature of its aggregation. The thermal unfolding analysis of the concentration gradient of TTPBgp12 and TFPgp17 indicates that with decreasing concentrations, both proteins increase in stability. However, a decrease in the protein concentration also causes an increase in its aggregation, for both TTPBgp12 and TFPgp17.
\end{abstract}

Keywords: tail tubular proteins; tail fiber proteins; biofilm

\section{Introduction}

Phage therapy is a promising alternative to antibiotics that are becoming increasingly less effective. Many phages that are effective against pathogenic strains have already been isolated. More than 95\% of all phages belong to the Caudovirales of order, which includes the Myoviridae, Podoviridae and Siphoviridae families [1]. The bacteriophage $\varphi \mathrm{O} 3-12$ belongs to the Podoviridae family and it is classified as part of the T7 group [2]. It was reported that most of the predicted gene products of phage $\varphi$ YeO3-12 are over $70 \%$ identical to those of the T3 genome. Therefore, the $\varphi$ YeO3-12 phage genome is extensively similar to the Enterobacteria T3 and T7 genomes [2].

Yersinia enterocolitica is a Gram-negative bacterium from the Enterobacteriaceae family. It is a widespread bacterium that can be found in soil and water environments. The reservoir of Yersinia 
enterocolitica serotypes is mainly found in pigs. People can become infected by eating uncooked pork contaminated with bacteria or through contact with the feces of livestock [3-6]. It is one of three known species of Yersinia, that is both pathogenic to humans and animals. Y. enterocolitica usually causes infections specific to the gastrointestinal track. Some common symptoms of infection are watery diarrhea, fever, vomiting, etc. [7,8].

The bacterial cell wall of Yersinia, as with other members of the whole Gram-negative bacteria group, contains an outer membrane in which the major component is lipopolysaccharide (LPS). LPS consists of three main parts: the lipid A, the core oligosaccharide that can often be divided into an inner and outer core, and an O-specific polysaccharide [9]. The presence of the complete LPS is required for the full virulence of Yersinia enterocolitica [10]. The outer core (OC) of the LPS is a particularly important virulence factor in Yersinia enterocolitica serotype O:3 (YeO3) [11].

The OC saccharide of YeO3 LPS contains 2-acetamido-2,6-dideoxy-D-xylo-hex-4-ulopyranose (Sugp), two glucose (Glc), one galactose (Gal) and two N-acetylgalactosamine (GalNAc) residues [9]. It was reported that the external part of the LPS also functions as a bacteriophage receptor $[9,12]$. Therefore, phages, that display specificity for a particular bacterial strain, could be used as potential diagnostic or therapeutic tools.

Phages attach to their host bacteria with the end of their tails. During infection, DNA is ejected from the capsid through a tail complex to the bacterial host. This stage is well characterized for the T7 phage [13]. The tail machine is composed of the following proteins: the portal (gp8), the adaptor (gp11), the nozzle (gp12, T7_TTPgp12) and the fiber (gp17, T7_TFPgp17). The portal protein, which exists in two conformations, acts as a valve on the portal pore. Thus, it is responsible for the regulation of the passage of DNA passage into the capsid. The adaptor protein interacts with the bottom of the portal protein, allowing DNA to slip along the tail up to the nozzle protein. The nozzle protein, T7_TTPgp12, contains four domains: the platform (interacting with the adaptor protein), the fiber dock interaction domain, the central $\beta$-propeller domain and the most distal nozzle tip domain. The nozzle, T7_TTPgp12, is the main protein responsible for closing and securing DNA inside the tail of the mature virus. The tail fiber protein, T7_TFPgp17, interacts with the bacterial LPS [14]. It has been reported that the C-terminal receptor-binding domain of T7_TTPgp17 is responsible for host-range determination, most likely by binding to a specific LPS region that varies between bacterial strains [15]. Additionally, it is well known that phages have evolved to be able to infect different hosts by adapting the receptor domain of TFP [16]. The adaptation is propelled by gene transfer, which accidentally leads to the acquisition new features. One example is the intramolecular chaperone (classified as S74 peptidase family) which contains a conserved C-terminal domain of an endosialidase found in the $\varphi \mathrm{K} 1 \mathrm{~F}$ phage. The gene encoding the chaperone has become a common feature of other TFPs and it can be removed by a self-cleaving mechanism $[17,18]$. The first $175 \mathrm{~N}$-terminal amino acid residues of this endosialidase show homology to the N-terminal regions of T7_TFPgp17, TFP of T3 phage (T3_TFPgp17) and TFP of $\varphi$ YeO3-12 phage [17]. The C-terminals of the TFPs differ since the C-terminals form the distal part of TFPs, which bind to the host receptors [19].

In our previous paper, we reported the biochemical features of TTPAgp11 of Yersinia phage $\varphi$ YeO3-12, which, in addition to its structural function, also acts as a sugar hydrolyzing enzyme [20]. In this paper, we are presenting our results of our bioinformatic analysis, biochemical characterization and antibacterial tests of two phage proteins. These proteins are the tail tubular protein B (TTPBgp12) and tail fiber protein (TFPgp17) originating from the Yersinia phage $\varphi$ YeO3-12. First, the nano differential scanning fluorimetry (nanoDSF) was used to assess the folding and stability of both proteins. Moreover, the stability in presence of different saccharides molecules was measured. 


\section{Materials and Methods}

\subsection{Gene Cloning and Protein Overexpression, Purification and Analysis}

The complete genome of bacteriophage $\varphi$ YeO3-12 was found in GenBank under accession number AJ251805. The genes that encode the tail tubular protein B (TTPB) and the tail fiber protein (TFP) (the gp12 and gp17), were amplified by polymerase chain reaction (PCR) using the following primers: TTPBgp12_FW, TACTTCCAATCCAATGCCATGCGCTCTTATGAGATGAAC and TTPBgp12_RV, TTATCCACTTCCAATGTTATTAGCGGTTAAGTAGACCAGAGG, TFPgp17_FW, TACTTCCAATCCAATGCCATGGCTACAACTATTAAGACCG and TFPgp17_RV, TTATCCACTT CCAATGTTACTAAGTCTTGTCCTTCTCCAAC. The genomic DNA was isolated from the phage lysate using a viral DNA extraction kit (Biocompare) and served as a template (100 ng) in the PCR reaction. A ligation-independent cloning method was used to clone the PCR products into the pMCSG9 vector using a T4 DNA polymerase [21]. Gene overexpression, protein isolation and purification were performed according to a slightly modified procedure described by Pyra et al. [20]. E. coli BL21(DE3)pLysS competent cells were used to overexpress both genes. The competent cells were added to Luria-Bertani (LB) medium with ampicillin, as well as chloramphenicol, in working concentrations of $100 \mu \mathrm{g} / \mathrm{mL}$ and $25 \mu \mathrm{g} / \mathrm{mL}$, respectively. Both proteins were purified using two rounds of nickel-affinity chromatography. Additionally, gel filtration using a Protein Arc 6-600 16/60 HR Sec column equilibrated with $50 \mathrm{mM}$ Tris/HCl buffer, pH 8.0, containing $300 \mathrm{mM} \mathrm{NaCl}, 5 \%$ glycerol and $1 \mathrm{mM}$ dithiothreitol, was used for purification. After the second nickel column, the flow-through fraction containing TTPBgp12 or TFPgp17 was precipitated using ammonium sulfate ( $95 \%$ saturation) overnight at $4{ }^{\circ} \mathrm{C}$. It was then pelleted by centrifugation, dissolved in the buffer mentioned above and then applied to a gel-filtration column. The TTPBgp12 or TFPgp17 fractions were collected and precipitated by ammonium sulfate (95\% saturation) and their purity was analyzed by $12 \%$ SDS-PAGE [22].

The secondary structure of both proteins was analyzed by circular dichroism (CD) spectroscopy. For the $\mathrm{CD}$ analysis, the proteins were dissolved in water with a final concentration of $\sim 7 \mu \mathrm{M}$. CD measurements were performed four times using a $1 \mathrm{~mm}$ cuvette. The measurements were taken in the wavelength range of 350 to $190 \mathrm{~nm}$ using a J-1000 Circular Dichroism spectrophotometer (Jasco Inc., Easton, MD, USA). These data were interpreted using the K2D3 method, which provided an estimation of the secondary structure of the proteins [23]. The proteins concentration was determined by the BCA method [24].

\subsection{Protein Folding and Aggregation Tests}

The thermal unfolding and protein aggregation experiments were carried out using a Prometheus instrument with nanoDSF and backreflection technology (NanoTemper, South San Francisco, CA, USA), using methods that were previously described [20].

NanoDSF technology allows for the automatic determination of thermal unfolding transition midpoints, $\operatorname{Tm}\left({ }^{\circ} \mathrm{C}\right)$. This technology also calculates the onset of unfolding using the transition midpoint and the slope of the unfolding signal. This method monitors unfolding-related tryptophan and tyrosine fluorescence at the emission wavelengths of $330 \mathrm{~nm}$ and $350 \mathrm{~nm}$, respectively.

The Prometheus device emits near-UV light at a wavelength that is scattered by aggregated proteins, which causes only non-scattered light to reach the detector. A direct measure of protein aggregation is the result of a reduction in backreflected light.

Both protein unfolding and aggregation tests were performed simultaneously. Then, $10 \mu \mathrm{L}$ of TTPBgp12 $(0.25 \mathrm{mg} / \mathrm{mL}, 2.78 \mu \mathrm{M})$ or TFPgp17 $(0.25 \mathrm{mg} / \mathrm{mL}, 3.6 \mu \mathrm{M})$ was placed in the capillary alone or mixed with one of the following sugars $(0.15 \mathrm{mg} / \mathrm{mL})$ : melibiose, galactose, glucose, maltose, $\beta$-lactose, $\alpha$-lactose, GalNAc and GlcNAc.

The samples were heated to $95^{\circ} \mathrm{C}$, and the fluorescence was monitored throughout the experiment. Data were analyzed with the PR.ThermControl and PR.StabilityAnalysis software packages (Publisher, South San Francisco, CA, USA) [25]. As for the results, melting scans of the studied proteins showed 
the following: the fluorescence ratio $(350 / 330 \mathrm{~nm})$ profile used to calculate $\mathrm{Tm}\left({ }^{\circ} \mathrm{C}\right)$, the first derivative profile showing the initial temperatures of unfolding, aggregation and the occurrence of conformational changes in the protein sample, as well as the scattering profile plotted as attenuation units (mAU) against temperature $\left({ }^{\circ} \mathrm{C}\right)$ indicating the onset temperature of protein aggregation.

\subsection{Measuring the Effect on Biofilm Formation}

One hundred microliters of inoculum, containing $2.2 \times 10^{7} \mathrm{CFU} / \mathrm{mL}$ of Yersinia enterocolitica O:3 was added to each well on 96-round well plates. Then, $10 \mu \mathrm{L}$ of $0.5 \mathrm{mM}$ of TTPBgp12 or TFPgp17 was added to the test samples. Ten microliters of lysis buffer $(300 \mathrm{mM} \mathrm{NaCl}, 20 \mathrm{mM}$ Tris- $\mathrm{HCl} \mathrm{pH}$ 8.0, 5\% glycerol, $5 \mathrm{mM} \mathrm{BME)}$, in which proteins were suspended, was added to the bacterial inoculum and considered as a negative control. As a positive control, $10 \mu \mathrm{L}$ of $10 \mathrm{mg} / \mathrm{mL}$ [26] gentamicin and $10 \mu \mathrm{L}$ of bacteriophage $\varphi$ Ye O3-12 $\left(4.5 \times 10^{7} \mathrm{PFU} / \mathrm{mL}\right)$ were used. All samples were triplicated. The 96-round well plates were incubated at $28^{\circ} \mathrm{C}$ for $24 \mathrm{~h}$. After incubation, the resulting biofilm was suspended in PBS and plated on agar plates. Agar plates were incubated at $28^{\circ} \mathrm{C}$ for $24 \mathrm{~h}$, after this time the number of colonies on the plates was counted. The bacteria layer was also placed on aLab-Tek ${ }^{\circledR}$ Chamber SlideTM System (Thermo Fisher Scientific Inc., Rochester, NY, USA), and incubated at $28^{\circ} \mathrm{C}$ for $24 \mathrm{~h}$. Then the slides were rinsed with water, dried, and stained with $1 \%$ crystal violet. After $30 \mathrm{~min}$, the slides were rinsed repeatedly with water and then observed under a light Olympus microscope (100× magnification).

\section{Results and Discussion}

\subsection{Bioinformatics Analysis}

While studying the proteins of bacteriophage tails, it was discovered that some structural tail tubular A proteins (TTPAs), of the environmental Klebsiella (KP32 and KP34) and Yersinia ( $\varphi$ YeO3-12) phages, can have dual-functionality $[20,27,28]$. These proteins were only previously considered to be structural phage tail proteins. Our results have shown that they exhibit a second function-a specific saccharide hydrolytic activity on biofilms formed by bacteria. This specificity can be used as an antibacterial agent with a wide spectrum of applications. So far, we have only examined TTPAs. The main question was whether other structural tail phage proteins, like tail tubular proteins B (TTPBs) or tail fiber proteins (TFPs), could also play an antibacterial role. The uncharacterized TTPB (UniProt code: Q9T105) and TFP (UniProt code: Q9T0Z9) encoded by gene 12 and 17, respectively, of Yersinia phage $\varphi$ YeO3-12 (GenBank code: AJ251805) were chosen for this study. Firstly, the primary structure of both selected proteins was analyzed using BLAST [29], Clustal Omega [30] and HHPred [31].

BLAST amino acid sequence analysis [29] of TTPBgp12 showed that it is similar to the TTPBs of phages that infect other types of bacteria with the highest similarity to bacteriophages infecting Yersinia, Escherichia, Salmonella, Citrobacter, Serratia, Klebsiella, Leclercia and Enterobacteria (97-99\%) (Supplementary data). Notably, amino acid sequence similarity was also found between TTPBgp12 and TFP from phages of Enterobacter (99-100\%). The observed amino acid sequence diversity is likely a reflection of the phage's adaptations in response to environmental changes and/or bacterial cell variability. Although a lot of proteins with high amino acid sequence similarity to TTPBgp12 were found, only one of them appeared to have been well characterized [13]. This protein was a tail tubular protein of Enterobacteria phage T7, T7_TTPgp12, which is a nozzle tail protein. This is the main protein responsible for closing and securing the DNA inside the tail, as well as phage absorption to the host bacteria outer membrane [13,32].

The Clustal Omega tool was used [24] to perform the amino acid sequence alignment of both proteins (Supplementary data) which allowed us to determine that TTPBgp12 is 69\% identical to T7_TTPgp12. 
The HHPred tool [31] confirmed a high similarity of both proteins predicting that TTPBgp12 is largely structurally homologous to T7_TTPgp12 with 100\% probability. This means that the overall fold of TTPBgp12 should be the same as in T7_TTPgp12.

The Phyre server [33] analysis showed that TTPBgp12 adopts probably a mainly $\beta$-structure (56\%) with only six very short $\alpha$-helical fragments (4\%) and some disordered regions (18\%). Domain analysis led to the finding of twenty short regions of polypeptide chain of TTPBgp12, which corresponds to some known domains of a different protein families. Although the confidence level does not specifically reflect the accuracy of the model, the level of confidence in the TTPBgp12 domain prediction was very low, about $20 \%$ or lower. Therefore, the conclusion was that there was no specific domain found in the structure of TTPBgp12.

The I-Tasser [34] server was used for further bioinformatic analysis of the 3D structure of TTPBgp12. The I-Tasser server modeled the structure by using the structures deposited in Protein Data bank (PDB) as a template. As a result, five three-dimensional models were generated. The structure of T7_TTPgp12, determined by cryo-electron microscopy (PDB code: 6r21), presented us with the best template for TTPBgp12 modelling (Z-score $>1$ ). The best obtained model of theTTPBgp12 structure suggests it adopts mainly a $\beta$-structure (Figure 1 ). In this prediction, all secondary structure elements matched very well between TTPBgp12 and T7_TTPgp12. This is not surprising, given the almost 70\% similarity of the amino acid sequence of both proteins. Interestingly, the only difference was seen in the one loop region which lacked two residues (R744-R745) in the T7_TTPgp12 structure. However, it modelled well in the corresponding loop of the predicted TTPBgp12 structure.

To build the 3D model for TTPBgp12, the Swiss-Model server [35] was used. Seven protein templates were found. Six of them were considered to be less suitable for modeling than the first one with the PDB code: 6r21. Thus, the only one model for TTPBgp12 was predicted. The obtained results were in accordance with TTPBgp12 structure predicted by the I-Tasser server. This was not surprising since both servers used the same protein template for model building.

In conclusion, the amino acid sequence and structure prediction analysis showed a high level of primary and tertiary structure similarity between TTPBgp12 and T7_TTPgp12. This suggests that the proteins have similar biochemical features and functions.

Based on the information deposited in the UniProt database, it is known that TFPgp17 contains a S74 peptidase domain at its C-terminus end (539-645 amino acid residues). This C-terminus end might be an intramolecular chaperone [17] removed after auto-proteolysis [18].

The BLAST analysis [29] showed that TFPgp17 had the highest amino acid similarity (99-100\%) to TFPs of other phages infecting Enterobacter, Yersinia, Serratia, Citrobacter, Shigella and Escherichia. There were also a lot of phage TFP amino acid sequences with a lower similarity found in the range of 40 to $90 \%$ (Supplementary data). No similarity was found between TFPgp17 and the TTPAs or the TTPBs. As for TTPBgp12, the amino acid sequence diversity among the TFPs is also reflective of the phage adaptations to environmental changes. Unfortunately, the most similar proteins to TFPgp17, in terms of their amino acid sequence, have not been studied. Therefore, we decided to align the amino acid sequence of TFPgp17 with amino acid sequences of only well biochemically or structurally characterized proteins. The following proteins were chosen for multiple protein sequence alignment analysis by BLAST [23] and Clustal Omega tool [24]: TFP of Enterobacteria phage T7 (T7_TFP), TFP of Enterobacteria phage T3 (T3_TFP) of tail spike protein of Enterobacteria phage K1F (K1F_TSP), L-shaped TFP of Escherichia phage T5 (T5_TFP), side TFP of prophage K12 (K12_TFP) and TFP of Enterobacteria phage lambda (L_TFP). The BLAST results revealed that most of the similarities in the amino acid sequences of the selected proteins were observed in the N-terminal regions of TFPgp17 and T7_TFP, as well as T3_TFP and K1F_TSP (Supplementary data). T5_TFP, K12_TFP and L_TFP only had very short fragments in their amino acid sequences that were similar to the polypeptide chain of TFPgp17, mainly present in the center and C-end regions (Supplementary data). To clarify, the Clustal Omega alignments were performed for two sets of amino acid sequences. The first input included the protein sequences with a higher BLAST alignment (TFPgp17 and T7_TFP, T3_TFP and K1F_TSP). The second 
set contained the protein sequences from the BLAST analysis with a smaller degree of similarity (TFPgp17 and T5_TFP, K12_TFP and L_TFP). All these analyses found that there is not a high similarity between the primary structures of TFPgp17 and the selected proteins, with similarity in the range of only $25 \%$ to $36 \%$ (Supplementary data).

$\mathbf{A}$

\section{Predicted TTPBgp12 structure}

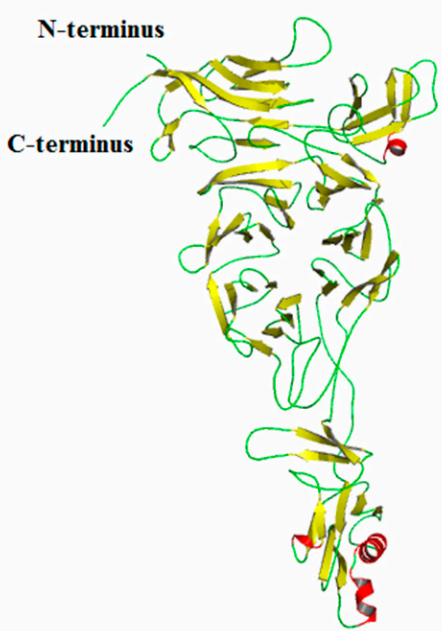

B

$$
\text { cryoEM T7_TTPgp12 structure }
$$

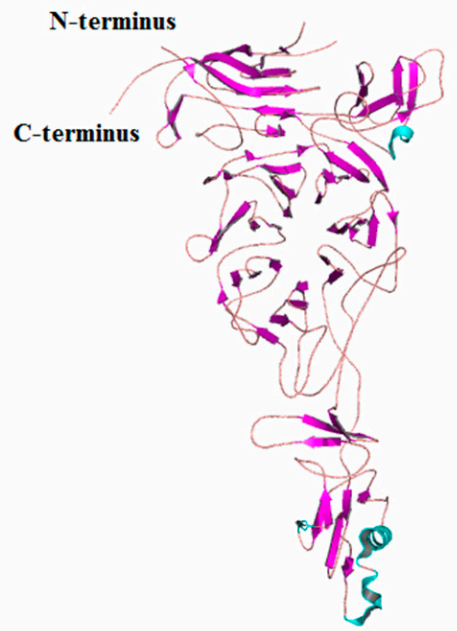

\section{$\mathrm{C}$}

Superimposition of TTPBgp12 and

T7_TTPBgp12 structures

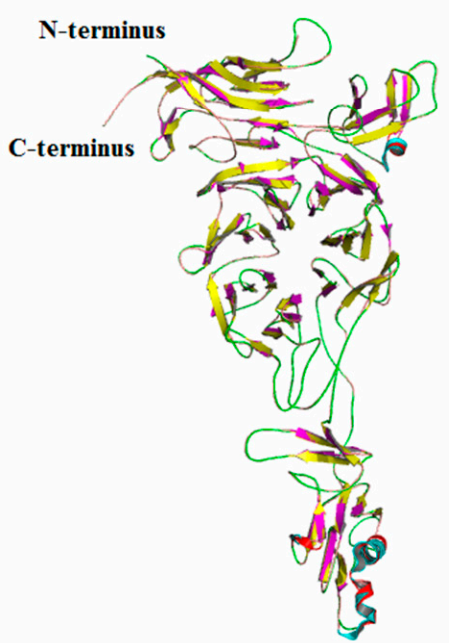

Figure 1. The predicted 3D structural model of tail tubular proteins B (TTPB)gp12 generated using the I-Tasser server [34]. (A) The presented structure was the best calculated model and was obtained using the cryoEM structure of T7_TTPgp12, (B) deposited in PDB (PDB code: 6r21). (C) The predicted structure of TTPBgp12 was superimposed onto cryoEM structure of the T7_TTPgp12 monomer.

Interestingly, the N-terminal of all the compared proteins was much more similar than the C-terminal region, even up to $\approx 78$. This was previously reported by Pajunen et al. [2]. This is in agreement with other findings that state that in many phages, the gene regions coding the C-terminus of TFPs evolve faster than other phage genes, as a result of intense host range selection [36]. This is due to the function of TFP that is responsible for binding to host cell receptors. Our analysis using the HHPred tool [31] also demonstrates a great diversity in the amino acid sequences of TFPs. The obtained results 
suggest that the polypeptide chain of TFPgp17 contains some structurally homologous fragments, $\sim 100$ to 200 amino acid residues long, to six phage proteins with a probability of 99\% (Supplementary data). These proteins include: endo- $N$-acetylneuraminidase (Enterobacteria phage K1F), receptor recognition protein (Salmonella phage vB_SenMS16), L-shaped tail fiber protein (Enterobacteria phage T5), neck appendage protein (Bacillus phage GA-1), phiAB6 tail spike (unidentified phage) and tail spike protein (Acinetobacter phage vB_AbaP_AS12). The homology of the rest of the hits from the HHPred analysis of TFPgp 17 was predicted with a probability of $\sim 60 \%$ and lower. Most of the listed proteins were structural tail phage proteins. The neck appendage protein of Bacillus phage GA-1 is classified as a chaperone, same as endo- $N$-acetylneuraminidase of Enterobacteria phage K1F, which additionally acts as hydrolase. That allows us to speculate that TFPgp17 is mainly a structural tail fiber protein. This protein could play the role of a chaperone protein, a receptor binding protein responsible for the bacterial cell recognition or could even exhibit hydrolytic activity.

The Phyre server analysis [33] found that the best protein templates for TFPgp17 domain prediction (with confidence 99\%) are in accordance with the results performed by the HHPred tool. These templates include the following: the neck appendage protein (Bacillus phage GA-1), the endo- $N$-acetylneuraminidase (Enterobacteria phage K1F) and the L-shaped tail fiber protein (Enterobacteria phage T5). All of the previously listed proteins represent chaperone proteins. The obtained results suggest that TFPgp17 could contain a chaperone domain on its C-terminus of its polypeptide chain. It is worth noting that the results obtained by both bioinformatic tools indicated that the acquired domains had a rather low percentage of identity, within the range of $13-24 \%$ for HHPred and $18-33 \%$ for Phyre analysis. The Phyre server analysis also discovered that TFPgp17 could contain an adhesion domain on its N-terminus and a confidence range of $90-96 \%$. The secondary structure and disorder prediction performed by the Phyre server showed that TFPgp17 probably contains more $\alpha$-helical elements (36\%) than $\beta$-strands (25\%). In addition, the disordered regions are on a similar level as the ordered helices ( $36 \%)$.

The I-Tasser server [34] was used to further analyze the spatial structure of TFPgp17. It generated five models of TFPgp17 based on the comparison to the protein structures deposited in the PDB. The best template proteins for modelling appeared to be the X-ray structures of endo- $N$-acetylneuraminidase of Enterobacteria phage K1F, (K1F_Nase, PDB code: 3gw6) [18] and the L-shaped tail fiber protein of Enterobacteria phage T5 (T5_TFP, PDB code: 4uw8) [36]. These two templates served as models for the prediction of the C-terminal of TFPgp17. The best obtained model of TFPgp17 structure suggests it mainly adopts a $\beta$-structure (Figure 2 ). In this prediction, only the C-terminal $\beta$-structure elements of TTPBgp12 and T5_TFP matched up well.

Sizeable differences were observed between the rest of the modeled TFPgp17 molecule and the templates proteins. This is not surprising, since the amino acid similarity of these regions, in TFPgp17 and T5_TFP, as well as K1F_Nase, is 15\% and 13\%, respectively. Therefore, we could assess that the overall structure of TFPgp17 predicted by the I-Tasser server was not correct. Moreover, this server has listed ten structural analogs in PDB to the predicted TFPgp17. The first required structure was a solid fit to the TFPgp17 model (Figure 2). That was the bacterial membrane protein RsaA with PDB code: $5 \mathrm{n} 8 \mathrm{p}$, which represents S-layer proteins [38]. This group of proteins includes a diverse class of molecules found in the surface layer (S-layer) of Gram-negative, Gram-positive bacteria and most archaea. The S-layer consists of repeating molecules of S-layer proteins that protect bacterial cells from the external environment, as well as play a role in pathogenicity. Based on this analysis, the following questions arise: Is it possible that the overall fold of TFPgp17 is actually similar to that of RsaA? Or, is it possible that, given the similar predicted structure of TFPgp17 to RsaA, could the phage protein exhibit similar features as have been seen with RsaA? Thus, could it build into the outer membrane of host bacterial cells, which would help the phage to infect bacteria. 
A

Predicted TFPgp17 structure

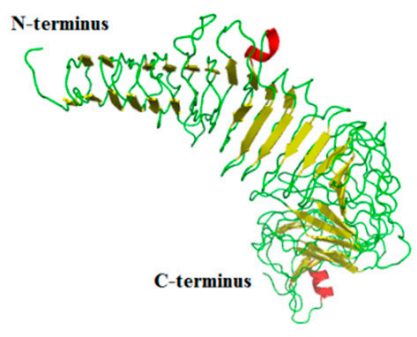

D

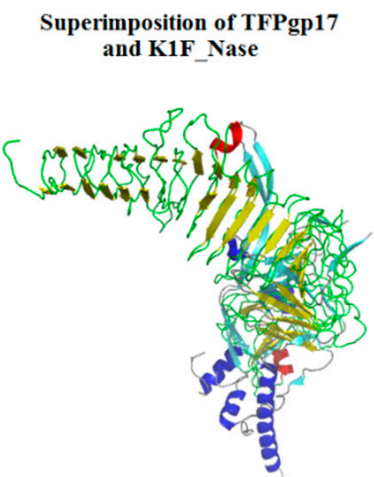

Crystal structure of RsaA

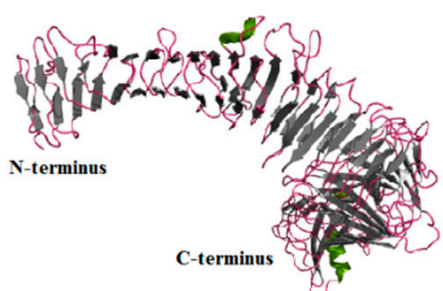

Crystal structure

of K1F Nase

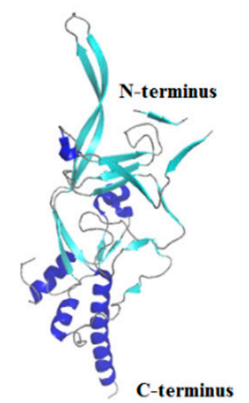

$\mathbf{E}$

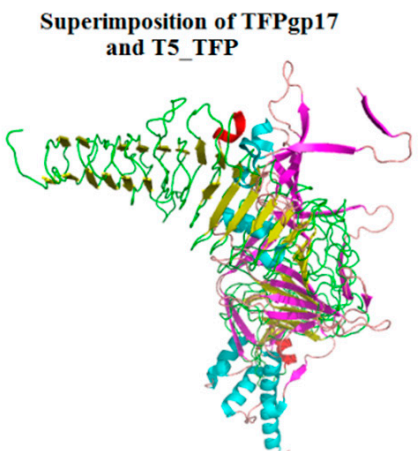

H

Superimposition of TFPgp 17 and RsaA

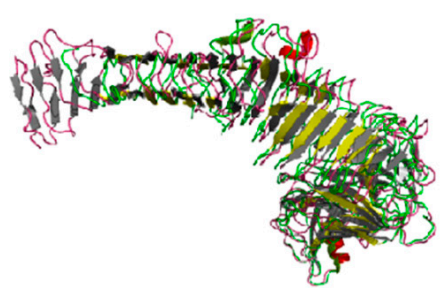

C

Crystal structure of

T5_TFP

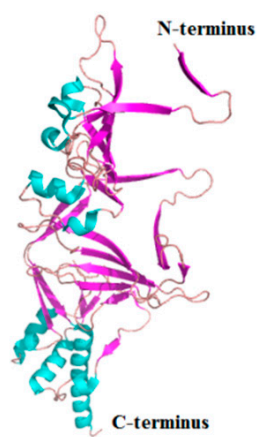

F

Superimposition of TFPgp17, K1F Nase and T5 TFP

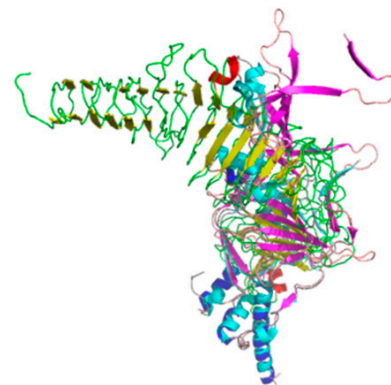

Figure 2. The predicted 3D structural model of TFPgp17 generated using the I-Tasser server [34]. (A) The presented structure was the best calculated model, and was obtained using the crystal structure of (B) endo- $N$-acetylneuraminidase of Enterobacteria phage $\varphi \mathrm{K} 1 \mathrm{~F}$, (K1F_Nase, PDB code: 3gw6) [18] and (C) L-shaped tail fiber protein of Enterobacteria phage T5 (T5_TFP, PDB code: 4uw8) [37]. The predicted structure of TTPBgp12 superimposed onto the crystal structure of K1F_Nase (D), T5_TFP (E) and both template proteins (F). (G) The crystal structure of RsaA (PDB code: 5n8p) [38], the structural analog of the predicted TFPgp17. (H) The predicted structure of TFPgp17 superimposed onto RsaA.

Another result from the Swiss-Model server presented with some interesting new information. In this study, 33 templates from PDB for TFPgp17 amino acid sequences were filtered. The templates with the highest quality, predicted from features of the target-template alignment, were selected for model building. The Swiss-Model server generated three partial models of the 3D TFPgp17 structure (Figure 3). 
A

N-terminal adhesin domain (residues 128-162)

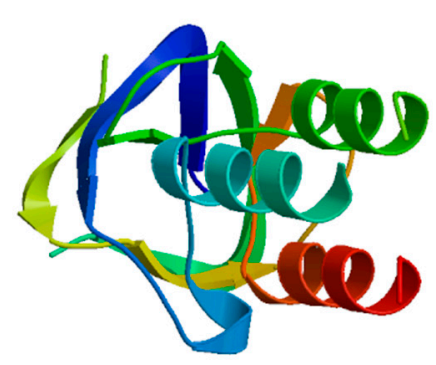

B

C-terminal $\mathrm{S} 74$ peptidase domain (residues 539-639)

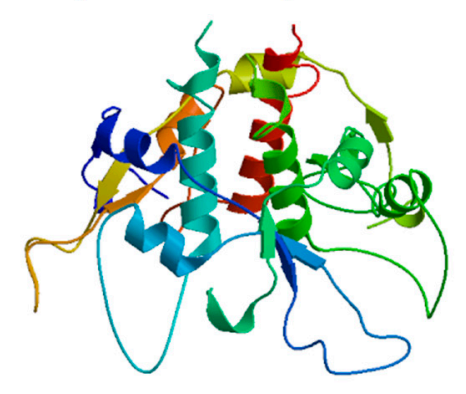

$\mathrm{C}$

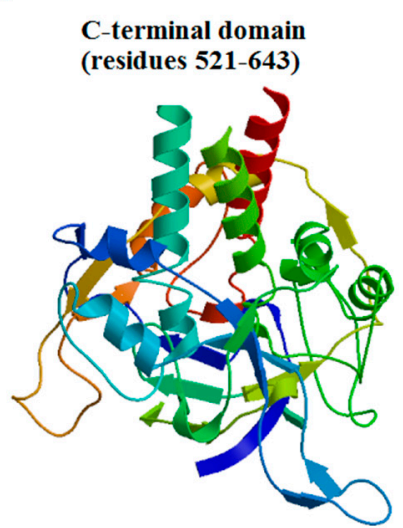

Figure 3. The predicted 3D partial structural models of tail fiber protein (TFP)gp17 generated using the Swiss-Model server [35]. (A) The model \#1-N-terminal region consisting of the amino acid residues 128-162 build on the template of adhesin A (PDB code: 3d9x) [39]. (B) The model \#2-the C-terminal S74 peptidase domain consisting of the amino acid residues 539-639 build on the template of the neck appendage protein (intramolecular chaperone) of Bacillus phage GA-1 (PDB code: 3gud) [18]. (C) The model \#3-C-terminal region consisting of the amino acid residues 521-643 build on the template of the L-shaped tail fiber protein of Enterobacteria phage T5 (PDB code: 4uw8) [37].

Model no. 1 was built on the template for adhesin A (PDB code: 3d9x) [39] and contains a fragment of the amino acid, containing residues 128-162, which adopts a mixed $\alpha / \beta$ secondary structure (37\% sequence similarity, 5\% coverage). The template for model no. 2 was a neck appendage protein (intramolecular chaperone) of Bacillus phage GA-1 (PDB code: 3gud) [18]. The predicted model includes the C-terminal S74 peptidase domain built of 539-640 amino acid residues (34\% sequence similarity, 14\% coverage). Additionally, the last 3D model of TFPgp17 was predicted for its C-terminal amino acid sequence (residues 521-643) using an L-shaped tail fiber protein from Enterobacteria phage T5 (T5_TFP, PDB code: 4uw8) [24] as a template (27\% sequence similarity, 16\% coverage). Models nos. 2 and 3 consist of a mixed $\alpha / \beta$ secondary structure, with an abundance of a helical structures.

In summary, all the bioinformatic tools used for the TFPgp17 amino acid sequence analysis allowed us to state that TFPgp17 undoubtedly belongs to the phage tail fiber protein family having an intramolecular chaperone domain at its C-terminal end. The N-terminal end of TFPgp17 showed a much higher similarity with another well-characterized representative of this protein family than its C-terminal regions. The great variability of the C-terminal regions is a common feature of TFPs due to being responsible for binding to the host receptors. The partially predicted TFPgp17 3D structure showed that this protein could possess an adhesion domain at the N-terminal region and an autoproteolytic S74 peptidase domain at the C-terminus of its polypeptide chain. The 3D structure of the whole TFPgp17 molecule was also predicted. It demonstrated that this protein could adopt an overall fold very similar to RsaA, which is reported as being a bacterial S-layer protein providing mechanical stability of the cell and protecting bacteria from the outside conditions. For a better characterization of biochemical features of TTPBgp12 and TFPgp17, experimental studies were carried out.

\subsection{Gene Cloning and Protein Overexpression, Purification and Analysis}

The genes for TTPBgp12 and TFPgp17 were cloned into a pMCSG9 vector and both overexpressed proteins were purified in two rounds of nickel-affinity chromatography. This was followed by gel filtration and analyzed with 12\% SDS-PAGE (Figures 4 and 5). 


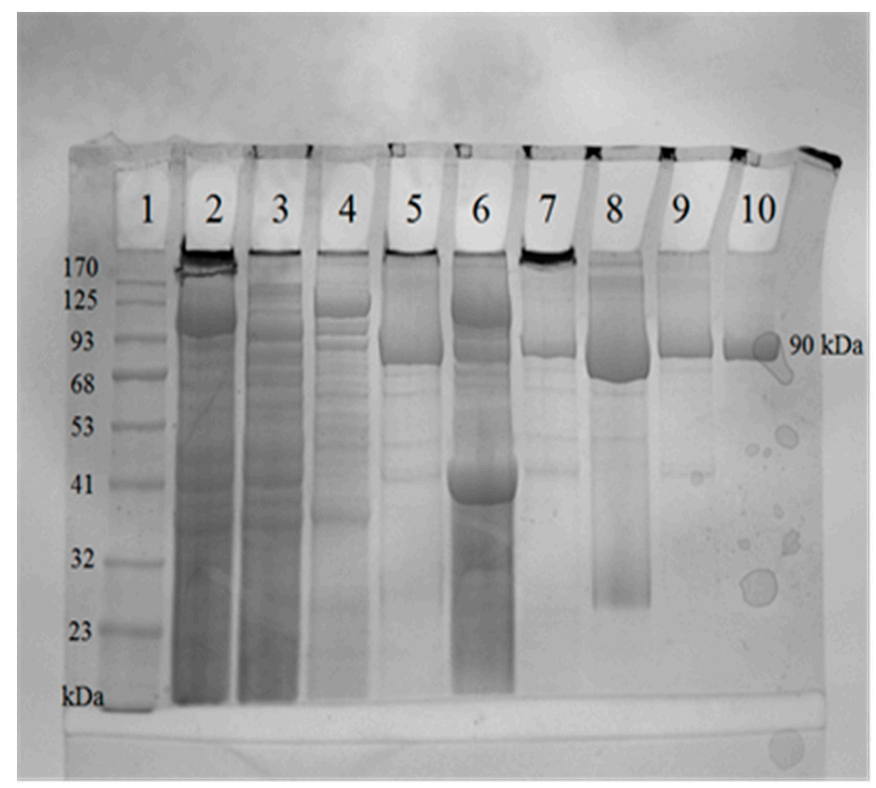

Figure 4. Analysis of TTPBgp12 from Yersinia phage $\varphi$ YeO3-12 using 12\% SDS-PAGE. The lanes are as follows: (1) prestained protein ladder-mid-range molecular weight (10-180 kDa) (Nippon Genetics); (2) crude extract; (3) the flow through fraction and (4) pooled fractions eluted with $250 \mathrm{mM}$ imidazole after the first round of nickel-immobilized affinity column; (5) TTPBgp12 fractions after Tobacco Etch Virus (TEV) protease cleavage; (6) pooled fractions eluted with $250 \mathrm{mM}$ imidazole after the second round of $\mathrm{Ni}^{2+}$-affinity chromatography; (7) denaturated and undenaturated (8) TTPBgp12 fractions after ammonium sulfate precipitation; (9) denaturated and undenaturated (10) TTPBgp12 solution after gel-filtration chromatography.

The SDS-PAGE showed that the purity of both proteins was over $90 \%$ and indicated that the molecular weight of TTPBgp12 was $90 \mathrm{kDa}$. This is in accordance with the theoretical value. It is interesting that the theoretical molecular mass of TFPgp17 is $69.44 \mathrm{kDa}$. However, based on the SDS-PAGE, the molecular weight was determined to be $\sim 58 \mathrm{kDa}$. This was not surprising, since TFPgp17 is a protein containing a S74 peptidase domain, which is responsible for protein autolysis. Hence, the molecular weight of the MBP-TFPgp17 fusion protein was $\sim 110 \mathrm{kDa}$. However, after TEV protease cleaved off the fusion protein and the second round of $\mathrm{Ni}^{2+}$-affinity chromatography, the molecular weight of TFPgp17 was indicated to be $~ 58 \mathrm{kDa}$ with SDS-PAGE. During this step of purification, a small protein, $\sim 11 \mathrm{kDa}$, was also observed in the gel. This corresponded to the molecular mass of the C-terminal S74 peptidase domain (539-645 amino acid residues).

\subsection{Protein Folding and Aggregation Tests}

The CD spectrum analysis (Figure 6) showed that both purified proteins were folded. They adopted a mixed $\alpha / \beta$ secondary structure with a large proportion of beta structure in TTPBgp12 and with similar content of helical and beta structures in TFPgp17. 

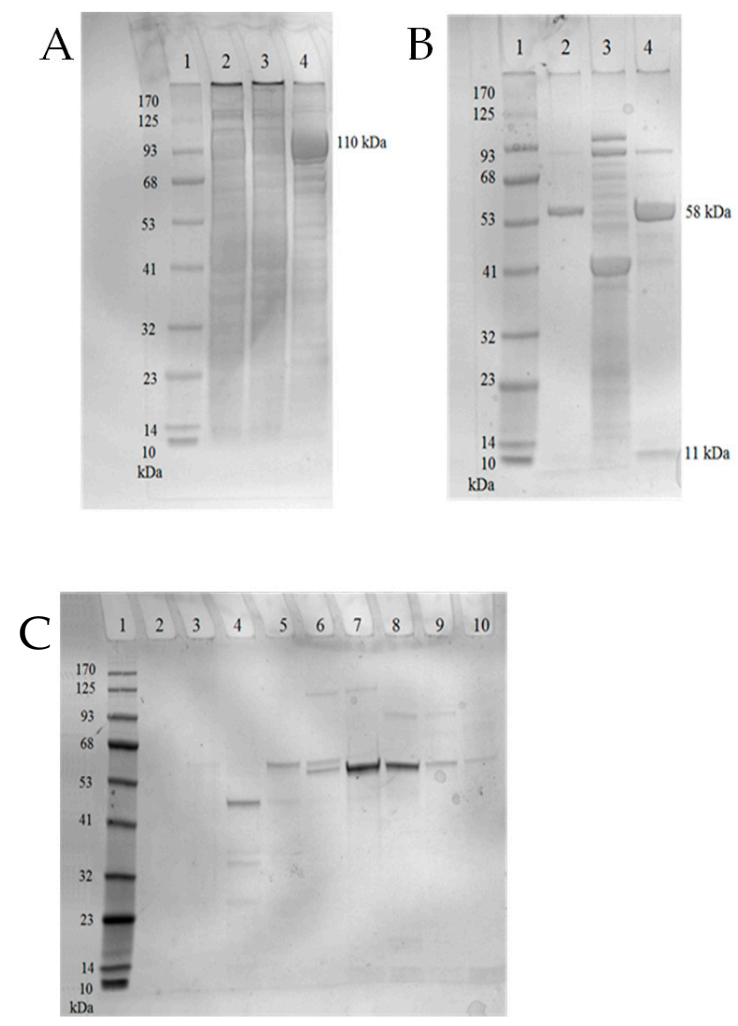

Figure 5. Analysis of TFPgp17 from Yersinia phage $\varphi$ YeO3-12 using 12\% SDS-PAGE. (A) The first round of nickel-immobilized affinity column. The lanes are as follows: (1) prestained protein ladder-mid-range molecular weight (10-170 kDa) (Nippon Genetics); (2) crude extract; (3) the flow through fraction; (4) pooled fractions eluted with $250 \mathrm{mM}$ imidazole. (B) The second round of nickel-immobilized affinity column. The lanes are as follows: (1) prestained protein ladder-mid-range molecular weight (10-170 kDa) (Abcam; (2) TFPgp17 fractions after TEV protease cleavage; (3) pooled fractions eluted with $250 \mathrm{mM}$ imidazole; (4) TFPgp17 fractions after ammonium sulfate precipitation. (C) Fractions obtained after gel-filtration chromatography: (1) mid-range molecular weight (10-170 kDa) (ABCAM), (2) collected fraction 1, (3) collected fraction 2, (4) collected fraction 3, (5) collected fraction 4, (6) collected fraction 5, (7) collected fraction 6, (8) collected fraction 7, (9) collected fraction 8, (10) collected fraction 9.

NanoDSF and backreflection technology was used to measure the stability, thermal unfolding and aggregation of TTPBgp12 and TFPgp17. Estimating the protein's stability is very important to researchers studying these macromolecules. This quantification reveals information about their thermal and colloidal features. Consequently, it is possible to determine the optimal conditions for large-scale production and long-term storage of proteins. We have also performed stabilization experiments for proteins in the presence of different mono- and disaccharides that are potential binders. In the experiment, the most popular saccharide moieties, which build bacterial polysaccharides components, were used. Some of them are components of the outer core of the lipopolysaccharide (LPS) of Y. enterocolitica such as GalNAc, glucose, galactose [40]. Increasing the stability of the proteins in the presence of saccharide molecules could be the premise for complex formation with the protein. However, the nanoDSF experiment is a preliminary screen test and cannot support whether the proteins can interact with sugars. Our results present differences in the stability and aggregation of TTPBgp12 and TFPgp17 (Figures 7 and 8, and Table 1). 
A)

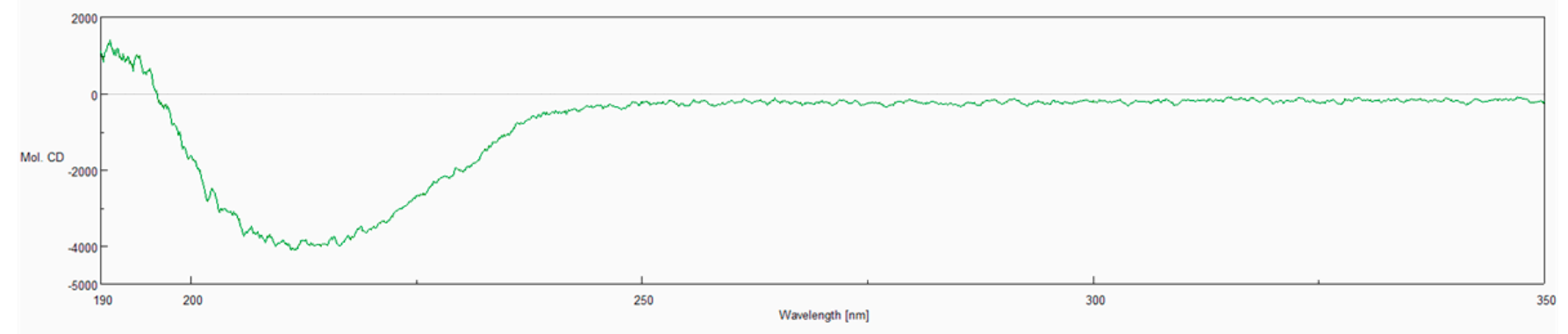

B)

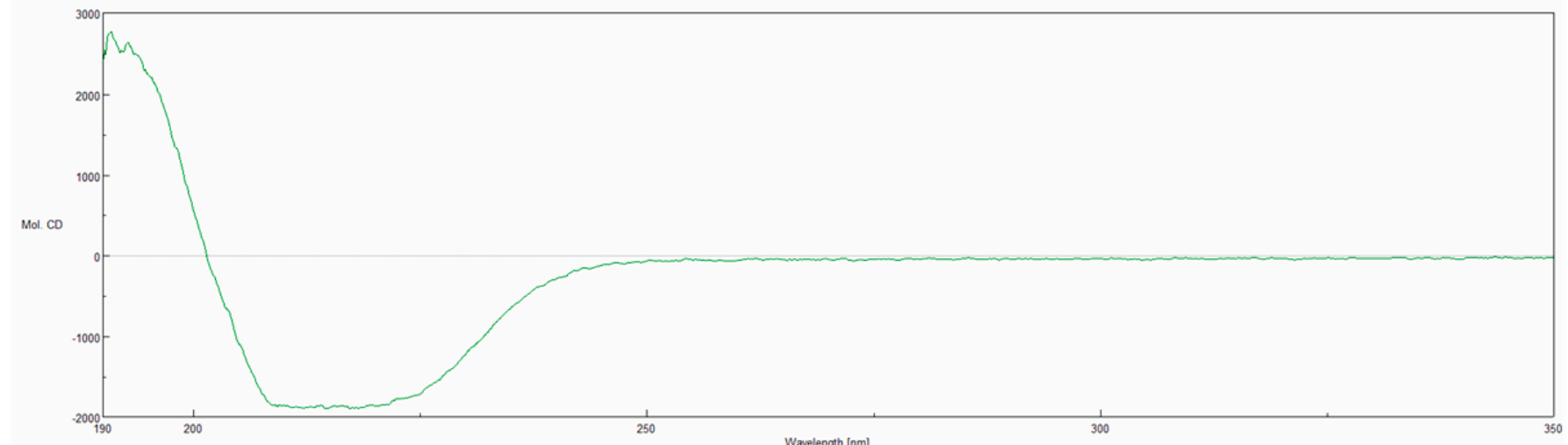

Figure 6. The circular dichroism (CD) spectrum of TTPBgp12 (A) and TFPgp17 (B) in units of molar ellipticity $\left(\mathrm{deg} \mathrm{cm}^{2} \mathrm{dmol}^{-1}\right)$. 

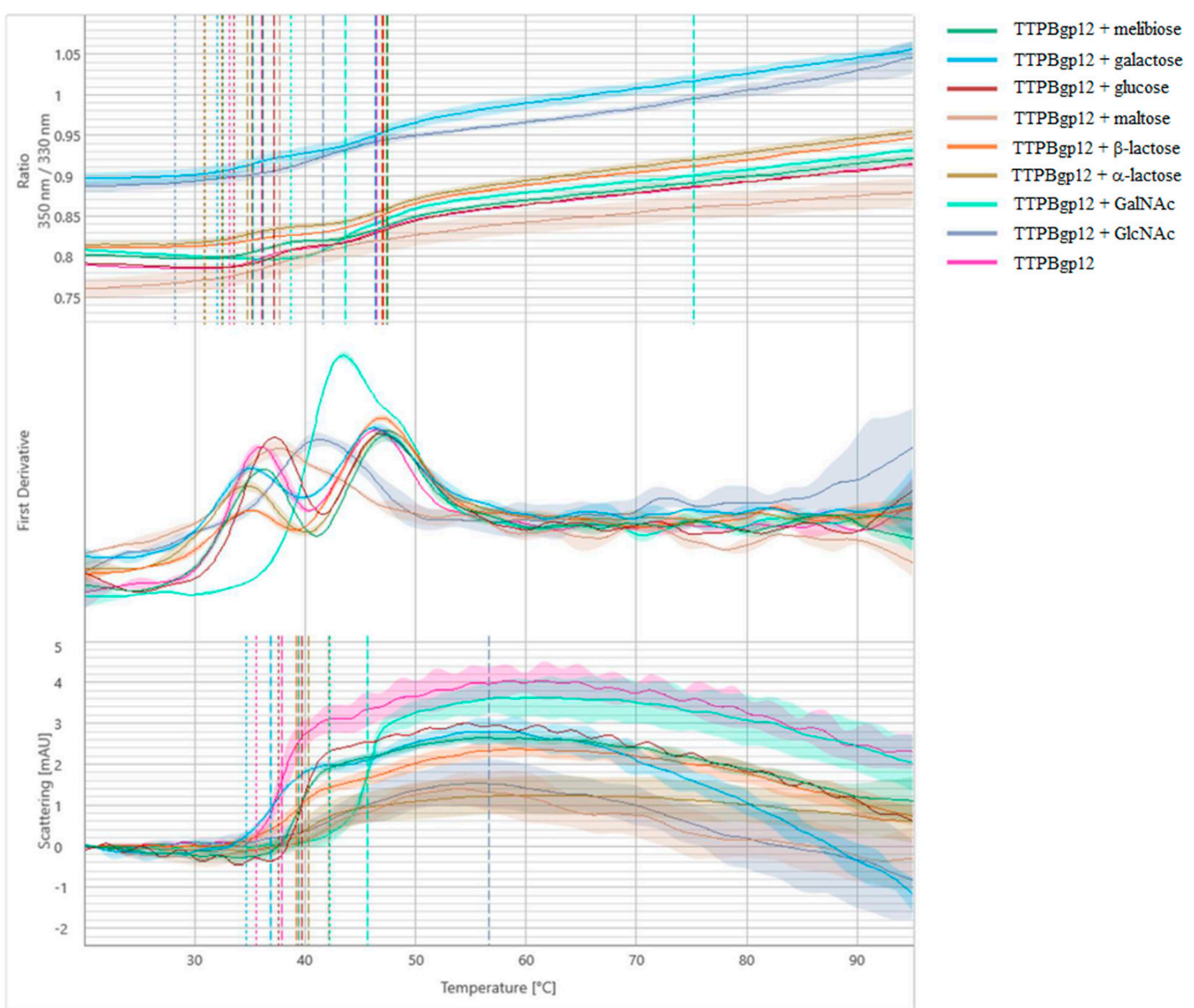

Figure 7. Melting analysis of TTPBgp12 with various sugars. The fluorescence ratio $(350 \mathrm{~nm} / 330 \mathrm{~nm})$ is shown in the top panel, the first derivative is shown in the middle panel and scattering is shown in the bottom panel. Thermal unfolding and aggregation onsets (indicated as the vertical dotted lines), as well as unfolding and aggregation transitions (indicated as the vertical dashed lines), are indicated by vertical lines in the graph. 

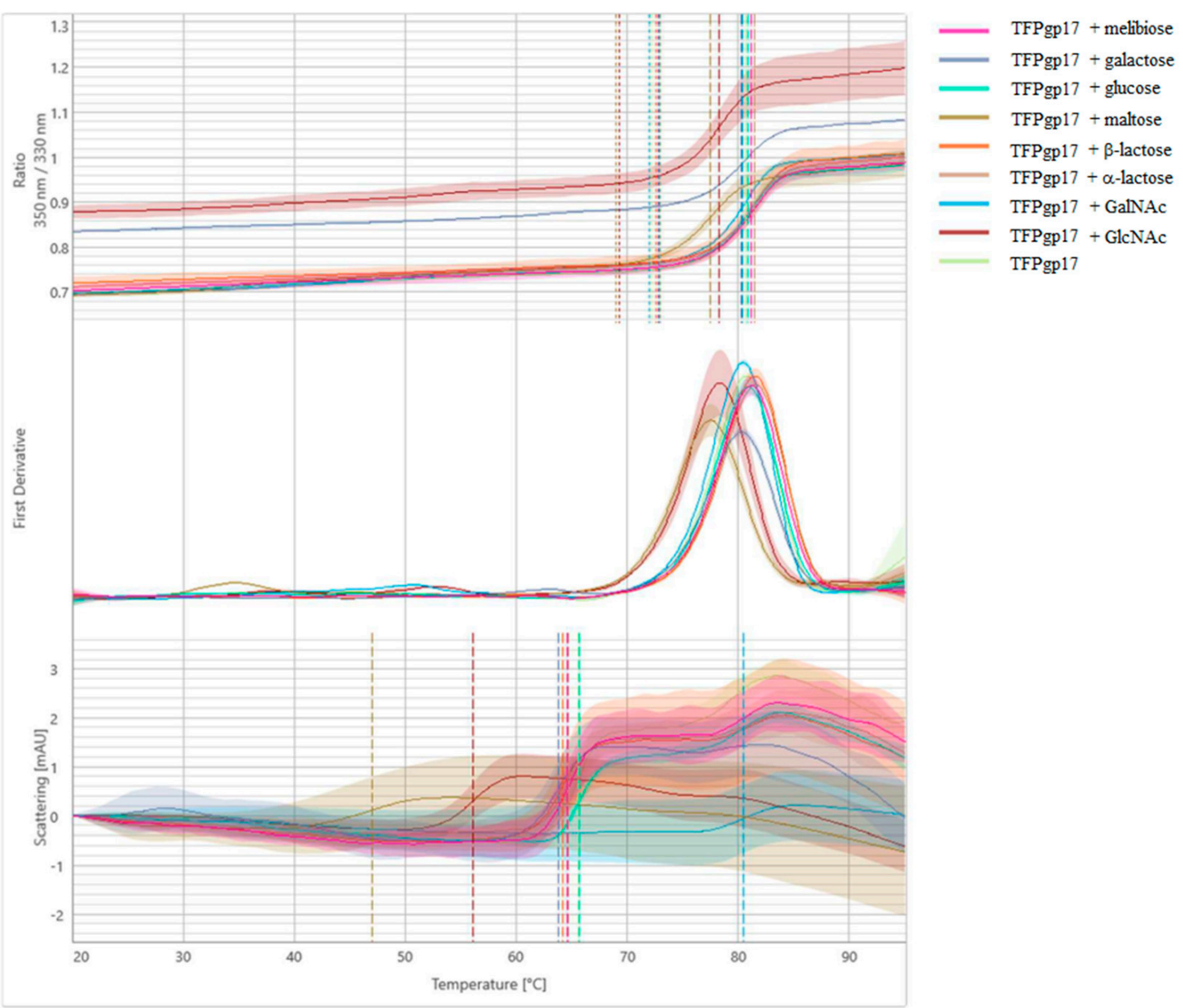

Figure 8. Melting analysis of TFPgp17 with various sugars. The fluorescence ratio $(350 \mathrm{~nm} / 330 \mathrm{~nm})$ is shown in the top panel, the first derivative is shown in the middle panel and scattering is shown in the bottom panel. Thermal unfolding and aggregation onsets (indicated as the vertical dotted lines), as well as unfolding and aggregation transitions (indicated as the vertical dashed lines), are indicated by vertical lines in the graph. 
Table 1. TTPBgp12 and TFPgp17 thermal unfolding and aggregation transitions midpoints $\left(\operatorname{Tm}\left({ }^{\circ} \mathrm{C}\right)\right)$ obtained from a melting scan of both proteins mixed with sugars, showing the fluorescence ratio $350 \mathrm{~nm} / 330 \mathrm{~nm}$ ), the first derivative onset and scattering.

\begin{tabular}{|c|c|c|c|c|}
\hline \multirow{3}{*}{ Sample } & \multicolumn{4}{|c|}{$\operatorname{Tm}\left({ }^{\circ} \mathrm{C}\right)$} \\
\hline & \multicolumn{2}{|c|}{$\begin{array}{l}\text { Fluorescence Ratio } \\
350 \mathrm{~nm} / 330 \mathrm{~nm}\end{array}$} & \multirow[t]{2}{*}{ First Derivative } & \multirow[t]{2}{*}{ Scattering } \\
\hline & $\# 1$ & $\# 2$ & & \\
\hline TTPBgp12/melibiose & 36.20 & 47.45 & 32.51 & 39.43 \\
\hline TTPBgp12/galactose & 35.30 & 46.40 & 32.02 & 36.93 \\
\hline TTPBgp12/glucose & 37.21 & 46.98 & NA & 33.76 \\
\hline TTPBgp12/maltose & 37.64 & NA & 30.85 & 42.11 \\
\hline TTPBgp12/ $\beta$-lactose & 35.13 & 46.96 & 32.46 & 39.19 \\
\hline TTPBgp $12 / \alpha$-lactose & 34.74 & 47.42 & 30.99 & 40.31 \\
\hline TTPBgp12/GalNAc & 43.65 & 75.19 & 38.73 & 45.65 \\
\hline TTPBgp12/GlcNAc & 41.66 & NA & 28.23 & 56.61 \\
\hline TTPBgp12 & 36.04 & 46.46 & 33.13 & 37.89 \\
\hline TFPgp17/melibiose & 81.16 & NA & 72.83 & 64.64 \\
\hline TFPgp17/galactose & 80.30 & NA & 72.88 & 63.78 \\
\hline TFPgp17/glucose & 80.86 & NA & 72.89 & 65.71 \\
\hline TFPgp17/maltose & 77.49 & NA & 69.03 & 46.99 \\
\hline TFPgp $17 / \beta$-lactose & 81.45 & NA & 72.57 & 64.19 \\
\hline TFPgp $17 / \alpha$-lactose & 81.51 & NA & 73.01 & 64.66 \\
\hline TFPgp17/GalNAc & 80.41 & NA & 71.96 & 80.45 \\
\hline TFPgp17/GlcNAc & 78.29 & NA & 69.28 & 56.06 \\
\hline TFPgp17 & 80.70 & NA & 72.15 & 65.55 \\
\hline
\end{tabular}

Each sample contained $0.25 \mathrm{mg} / \mathrm{mL}$ of the tail protein and $0.15 \mathrm{mg} / \mathrm{mL}$ of the sugar.

TTPBgp12 was shown to have two unfolding events (at $\sim 36$ and $46^{\circ} \mathrm{C}$ ), suggesting that the protein is a dimer. We have observed that sugars overall were shown to have a different effect on the general stability of TTPBgp12, which aggregated in the presence of all the tested sugars. Interestingly, GlcNAc and GalNAc significantly increased the temperature at which the aggregation was detected, to $~ 56$ and $\sim 45^{\circ} \mathrm{C}$, by GlcNAc and GalNAc, respectively. It is worth noting that maltose and GlcNAc shift two unfolding events to one global unfolding. Interestingly, in the crystal structure of T7_TTPgp12 (the structural homolog of TTPBgp12), four domains were determined [13]. If we assume that TTPBgp12 adopts the spatial structure very similar to T7_TTPgp12, then it is possible that some domains unfold at $\sim 36$ and other domains unfold at $\sim 46^{\circ} \mathrm{C}$. We think that the nozzle tip domain is the most distal and thus a separate domain. Therefore, it could unfold independently of the other domains.

TFPgp17 was shown to have a higher stability than TTPBgp12 with the one unfolding event at $\sim 80^{\circ} \mathrm{C}$. Interestingly, the aggregation of TFPgp 17 precedes the main unfolding event (at $\sim 65^{\circ} \mathrm{C}$ ). It was observed that sugars can play various roles in the overall stability of TFPgp17. Among the sugars tested, maltose and GlcNAc were shown to have the highest effect on TFPgp17 aggregation. The sugars caused a large decrease in the observed temperature, to $\sim 47$ and $\sim 56^{\circ} \mathrm{C}$, by maltose and GlcNAc, respectively. The presence of GalNAc increased the temperature of aggregation to $\sim 80^{\circ} \mathrm{C}$, leading to the protein unfolding and aggregation events at the same time. NanoDSF and backreflection technology allowed us to determine the effect of the protein concentration on its stability (Figures 9 and 10 and Table 2). The analysis of thermal unfolding profiles of the concentration gradient of TTPBgp12 and TFPgp17 clearly showed that with decreasing concentrations, both proteins increased in stability. As previously mentioned, the unfolding of individual domains of TTPBgp12 can even be detected at a concentration of $\sim 0.25 \mathrm{mg} / \mathrm{mL}$ and higher. It is worth noting, that at the same time, a decrease in protein concentration can cause an increase in aggregation, for both TTPBgp12 and TFPgp17. These inverted patterns suggest that the optimization of the amount of protein in the buffer solution might help increase purification yield of the protein. It is more prone to aggregate when diluted, which is 
the same condition presented during the flow through the chromatography columns. Additionally, for long-term storage it might be more optimal to lower the aggregation under unfolded conditions and establish refolding conditions to keep higher concentrations of functional folded protein in the experimental assays.

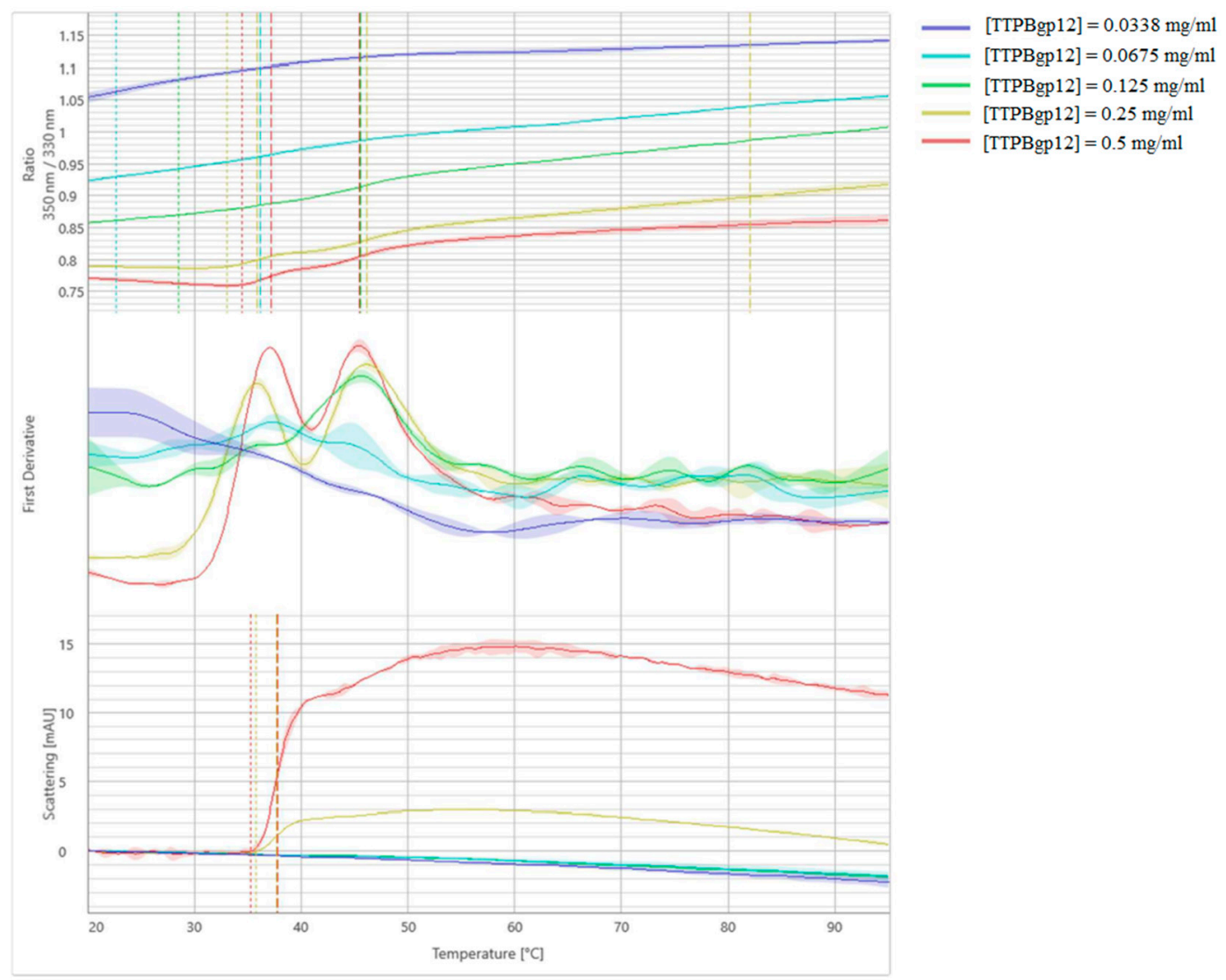

Figure 9. Melting analysis of the concentration gradient of TTPBgp12. The fluorescence ratio $(350 \mathrm{~nm} / 330 \mathrm{~nm})$ is shown in the top panel, the first derivative is shown in the middle panel and scattering is shown in the bottom panel. Thermal unfolding and aggregation onsets (indicated as the vertical dotted lines), as well as unfolding and aggregation transitions (indicated as the vertical dashed lines), are indicated by vertical lines in the graph.

Table 2. TTPBgp12 and TFPgp17 thermal unfolding and aggregation transitions midpoints $\left(\operatorname{Tm}\left({ }^{\circ} \mathrm{C}\right)\right)$ obtained from a melting scan of concentration gradient of both proteins, showing the fluorescence ratio $(350 \mathrm{~nm} / 330 \mathrm{~nm})$, the first derivative onset and scattering.

\begin{tabular}{|c|c|c|c|c|c|}
\hline \multirow[b]{3}{*}{ Protein } & \multirow{3}{*}{$\begin{array}{l}\text { Sample } \\
\text { Concentration }(\mathrm{mg} / \mathrm{mL})\end{array}$} & \multicolumn{4}{|c|}{$\operatorname{Tm}\left({ }^{\circ} \mathrm{C}\right)$} \\
\hline & & \multicolumn{2}{|c|}{$\begin{array}{l}\text { Fluorescence Ratio } \\
350 \mathrm{~nm} / 330 \mathrm{~nm}\end{array}$} & \multirow{2}{*}{ First Derivative } & \multirow{2}{*}{ Scattering } \\
\hline & & $\# 1$ & $\# 2$ & & \\
\hline \multirow{5}{*}{ TTPBgp12 } & 0.0338 & NA & NA & NA & NA \\
\hline & 0.0675 & NA & NA & NA & NA \\
\hline & 0.125 & 45.58 & NA & 28.50 & NA \\
\hline & 0.25 & 35.87 & 46.13 & 33.03 & 37.68 \\
\hline & 0.5 & 37.17 & 45.46 & 34.46 & 37.79 \\
\hline \multirow{5}{*}{ TFPgp17 } & 0.0338 & 71.30 & NA & 59.45 & NA \\
\hline & 0.0675 & 76.80 & NA & 66.78 & NA \\
\hline & 0.125 & 79.70 & NA & 72.49 & NA \\
\hline & 0.25 & 80.54 & NA & 72.18 & 65.34 \\
\hline & 0.5 & 80.67 & NA & 72.24 & 64.50 \\
\hline
\end{tabular}




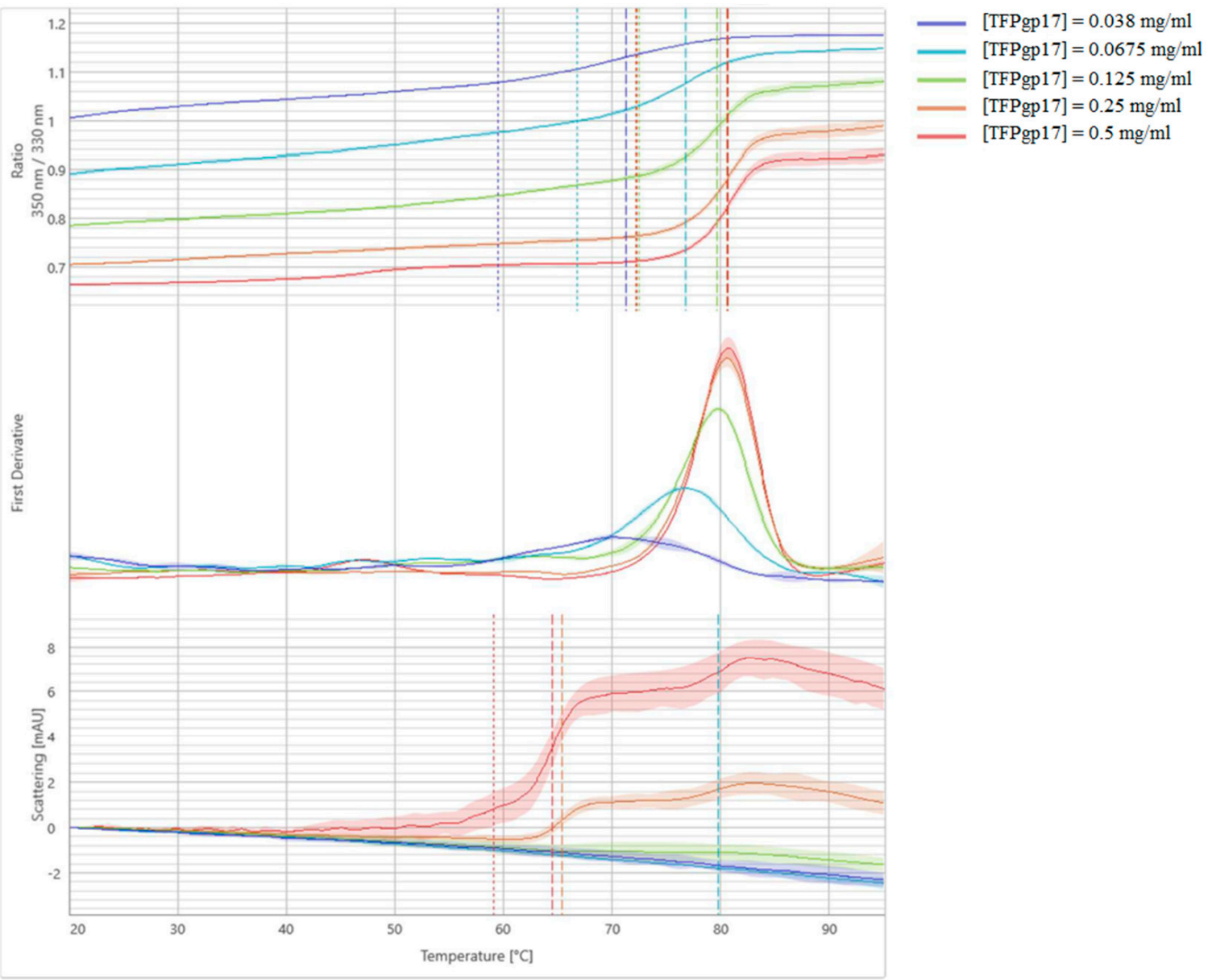

Figure 10. Melting analysis of the concentration gradient of TFPgp17. The fluorescence ratio $(350 \mathrm{~nm} / 330 \mathrm{~nm})$ is shown in the top panel, the first derivative is shown in the middle panel and scattering is shown in the bottom panel. Thermal unfolding and aggregation onsets (indicated as the vertical dotted lines), as well as unfolding and aggregation transitions (indicated as the vertical dashed lines), are indicated by vertical lines in the graph.

\subsection{Influence of TTPBgp12 and TFPgp17 on Biofilm Formation}

As previously mentioned, tail phage proteins display a structural function, but they can play additional biological roles as well $[20,27,28]$. The purified TTPBgp12 and TFPgp17 were used in experiments regarding their effect on the bacterial Yersinia enterocolitica $\mathrm{O}: 3$ cells growth and biofilm formation. During bacteria incubation, TTPBgp12 or TFPgp17 was shown to have an inhibiting effect on bacterial culture growth. The effect was visible both in the bacterial suspension and during biofilm formation compared to the control samples (Figure 11). 


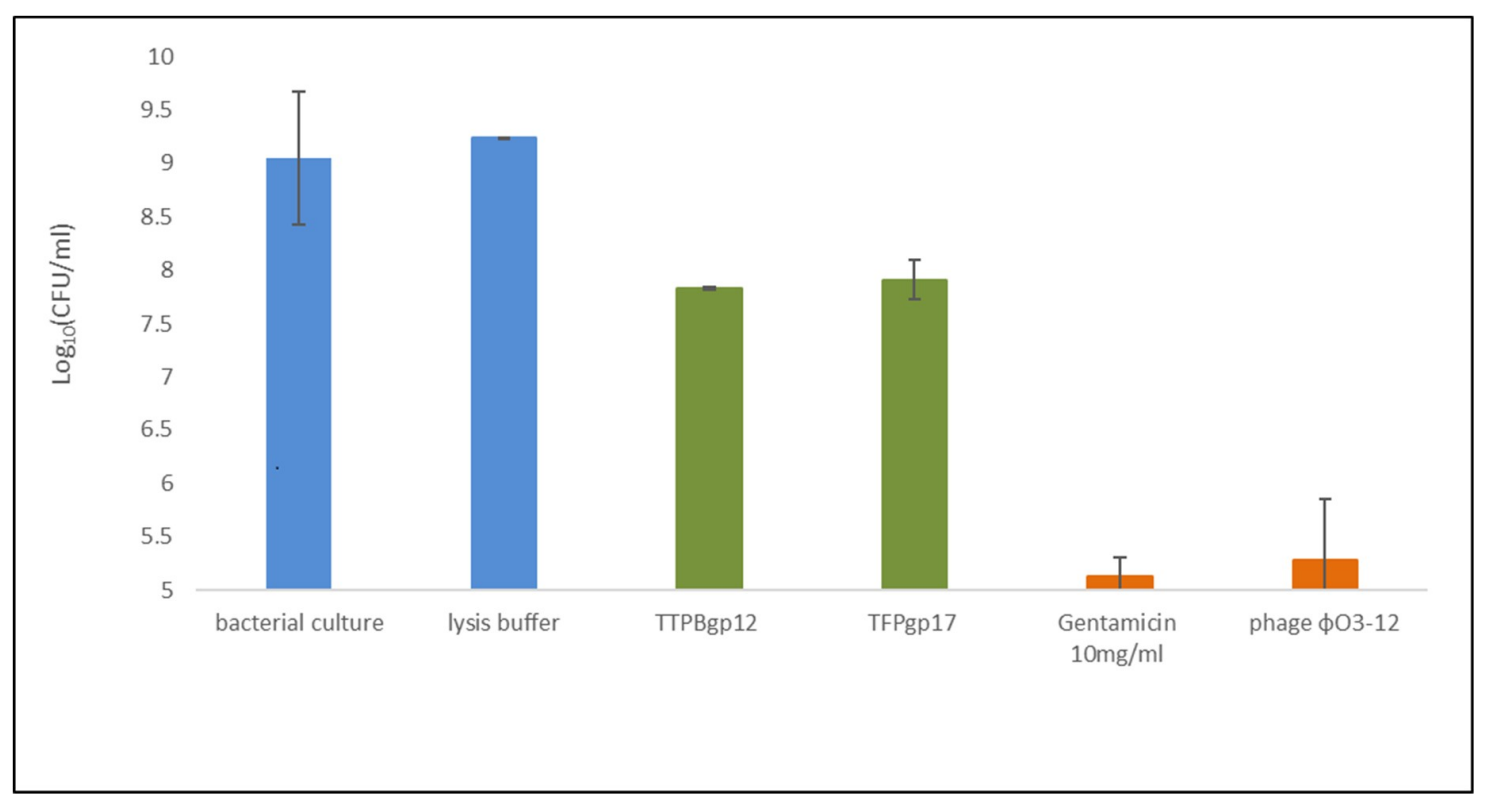

Figure 11. The effect of TTPBgp12 and TFPgp17 (in green) on Yersinia enterocolitica O:3 growth and the biofilm formation. The negative control (in blue) was lysis buffer (buffer in which proteins were suspended during the test) and the positive control (in orange) were gentamicin $(10 \mathrm{mg} / \mathrm{mL}$ ) and phage $\varphi$ YeO3-12. The untreated bacterial culture was shown in yellow. All results were presented as averages of results from three independent replicates in three parallel trials. Error bars represent the means, standard deviations. CFU, colony-forming unit.

Moreover, the visualization of biofilm layers stained with crystal violet confirmed this observation (Figure 12).
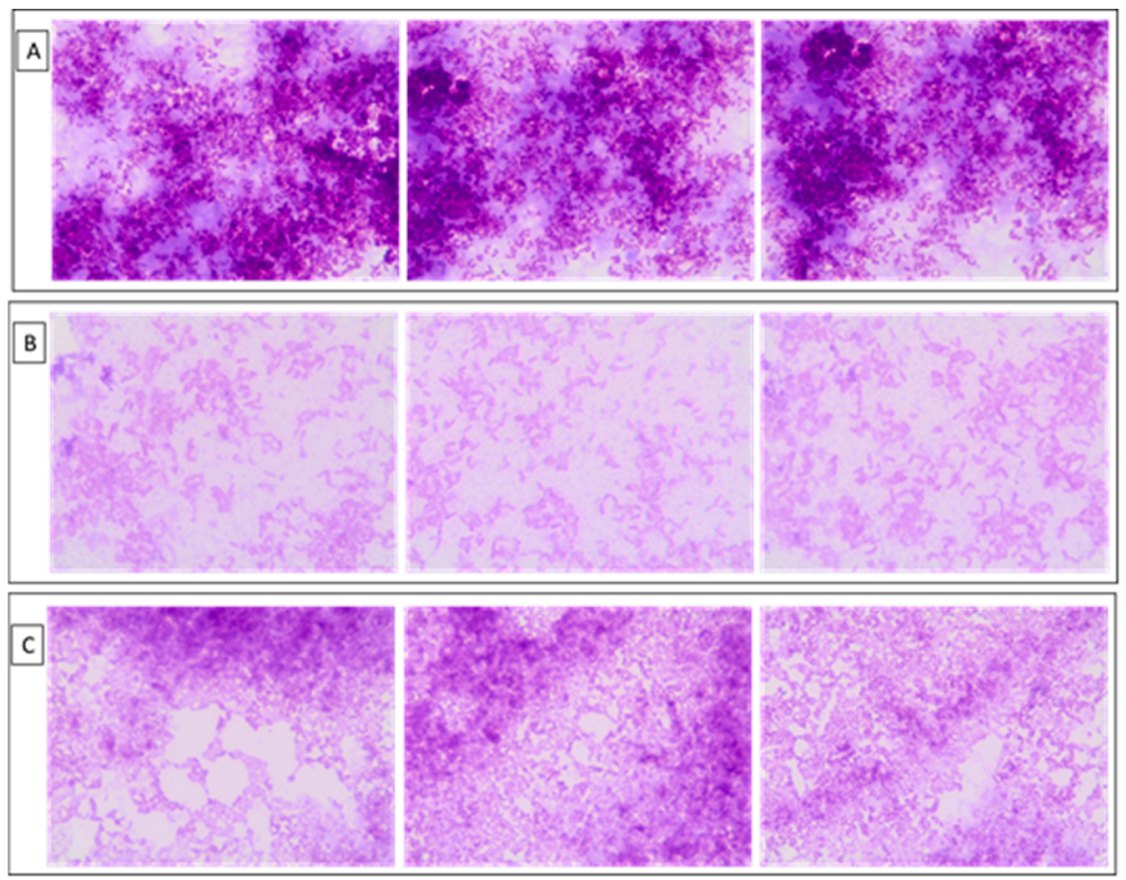

Figure 12. The effect of TTPBgp12 and TFPgp17 on the biofilm formation observed in a light microscope Olympus BX43 (magnitude 100×). The biofilm formed by Yersinia enterocolitica O:3 was stained with 1\% crystal violet. (A) The control sample (Yersinia enterocolitica O:3 alone). (B) The bacteria incubated with TTPBgp12. (C) The bacteria incubated with TFPgp17. 
The obtained results showed that both TTPBgp12 and TFPgp17 were able to inhibit the growth of bacteria and could influence biofilm formation. This observation could open a wide field for further research towards explaining the mechanism of this phenomenon. In natural conditions, both TTPBgp12 and TFPgp17 were the part of the tail phage machinery involved in bacterial cells infection. The role of TTPBgp12 is still undetermined. However, it was shown that this protein can bind to bacterial cells after specific recognition by TFP. TTPBgp12 can limit bacterial communication with the external environment, decreasing the flow between the inside and the outside of the bacteria. In contrast, TFPgp17 was observed to be responsible for recognition and binding to the host receptors. It is most likely that the binding is reversible until the TTPAgp11 and TTPBgp12 come into action. Moreover, the bioinformatics analysis allows us to speculate that TFPgp17 itself could even be incorporated into the bacterial cell wall, resulting in the formation of pores. This could increase the cell membrane permeability of bacteria, efflux of a substances, and consequently even bacterial cell death. For now, these are our assumptions. Further research will allow us to find out the answers to our questions.

\section{Conclusions}

The goal of this work was to characterize the biochemical, functional structure, as well as the antibacterial activity of new members of the tail tubular protein B (TTPB) and tail fiber protein (TFP) families. We have chosen TTPBgp12 and TFPgp17, previously classified as tail phage proteins. The UniProt database was lacking on the information of the transcript, and homology levels of these proteins. Bioinformatic tools were used to analyze the similarity of the selected proteins to other members of their families. TTPBgp12 showed a high similarity of primary $(69 \%)$ and spatial structure to the tail tubular protein of Enterobacteria phage T7.

TFPgp17 did not display a high similarity to other known TFPs. However, its N-terminal amino acid sequence is much more similar to corresponding fragments of other TFPs than its C-terminal regions. The C-terminus of TFPgp17 contains an intramolecular chaperone domain, which is common among the TFP family. The overall fold of the predicted 3D structure of TFPgp17 is very similar to S-layer proteins responsible for the stability of the bacteria cells.

For the first time, the genes of both proteins were overexpressed in E. coli cells and the proteins were purified by nickel-affinity and gel-filtration chromatography. The thermal unfolding experiments showed that TTPBgp12 is a two-domain protein in contrast to the single-domain protein, TFPgp17. TFPgp17 is a much more stable protein than TTPBgp12 which is indicated by the unfolding temperature of $\sim 80{ }^{\circ} \mathrm{C}$ for TFPgp17, while the unfolding temperatures for the domains in TTPBgp12 are $\sim 36$ and $46^{\circ} \mathrm{C}$.

The presence of sugars affects the stability of both proteins causing their aggregation. It has also been observed that the stability of TTPBgp12 and TFPgp17 increases when of their concentrations are decreased. However, decreasing the protein concentration causes an increase in its aggregation, both for TTPBgp12 and TFPgp17. The incubation of TTPBgp12 or TFPgp17 with Yersinia enterocolitica O:3 inhibited bacterial cell growth, as well as impacted the biofilm formation. These results revealed the antibacterial activity of both tested phage proteins. The mechanism of this activity is not yet known. We hypothesize that TTPBgp12, which acts as an adhesive, may limit the uptake of nutrients in bacteria culture. TFPgp17, similar to other S-layer proteins, can form pores in the bacterial membrane, resulting in an increase in permeabilization and transport interruption. More studies are needed to further understand the structure and function of TTPBgp12 and TFPgp17. Our results suggest that both TTPBgp12 and TFPgp17 could potentially be used as a biofilm inhibiting factor.

Supplementary Materials: The following are available online: the BLAST amino acid sequence analysis, the HHpred analysis. 
Author Contributions: A.P.: conceptualization, methodology, results analysis, writing and editing; E.B.: conceptualization, methodology, results analysis, writing and editing, revision, supervision and funding acquisition; K.F.: methodology, results analysis, experiments conduction, revision; B.S.-O.: methodology, experiment conduction, results analysis, revision; A.C.: experiment conduction, results analysis. All authors have read and agreed to the published version of the manuscript.

Funding: This research was funded by the National Science Center (Poland), grant number 2017/26/E/NZ1/00249.

Acknowledgments: We would like to thank Mikael Skurnik for providing the phage, Piotr Chmielewski for providing access to the CD spectrophotometer, Katherine Tyrlik for language correction, and the NanoTemper Company for allowing us to perform protein folding and aggregation measurements.

Conflicts of Interest: The authors declare no conflict of interest.

\section{References}

1. Ackermann, H.W.; Prangishvili, D. Prokaryote viruses studied by electron microscopy. Arch. Virol. 2012, 157, 1843-1849. [CrossRef] [PubMed]

2. Pajunen, M.I.; Kiljunen, S.J.; Söderholm, M.E.; Skurnik, M. Complete genomic sequence of the lytic bacteriophage phiYeO3-12 of Yersinia enterocolitica Serotype O:3. J. Bacteriol. 2001, 183, 1928-1937. [CrossRef] [PubMed]

3. Bottone, E.J. Yersinia enterocolitica: The charisma continues. Clin. Microbiol. Rev. 1997, 10, $257-276$. [CrossRef]

4. Bottone, E.J. Yersinia enterocolitica: Overview and epidemiologic correlates. Clin. Microbes. Infect. 1999, 1, 323-333. [CrossRef]

5. Sabina, Y.; Rahman, A.; Ray, R.C.; Montet, D. Yersinia enterocolitica: Mode of transmission, molecular insights of virulence, and pathogenesis of infection. J. Pathog. 2011. [CrossRef]

6. Wang, X.; Li, Y.; Jing, H.; Ren, Y.; Zhou, Z.; Wang, S.; Wang, L. Complete genome sequence of a Yersinia enterocolitica "Old World"(3/O: 9) strain and comparison with the "New World"(1B/O: 8) strain. J. Clin. Microbiol. 2011, 49, 1251-1259. [CrossRef]

7. Cover, T.L.; Aber, R.C. Yersinia enterocolitica. N. Engl. J. Med. 1987, 321, 16-24. [CrossRef]

8. Fàbrega, A.; Vila, J. Yersinia enterocolitica: Pathogenesis, virulence and antimicrobial resistance. Enfermedades Infecciosas Microbiologia Clinica 2012, 30, 24-32. [CrossRef] [PubMed]

9. Pinta, E.; Duda, K.A.; Hanuszkiewicz, A.; Kaczyński, Z.; Lindner, B.; Miller, W.L.; Hyytiäinen, H.; Vogel, C.; Borowski, S.; Kasperkiewicz, K.; et al. Identification and role of a 6-deoxy-4-keto-hexosamine in the lipopolysaccharide outer core of Yersinia enterocolitica serotype O:3. Chem. A Eur. J. 2009, 15, 9747-9754. [CrossRef]

10. al-Hendy, A.; Toivanen, P.; Skurnik, M. Lipopolysaccharide O side chain of Yersinia enterocolitica O:3 is an essental virulence factor in an orally infected murine model. Infect. Immun. 1992, 60, 870-875. [CrossRef] [PubMed]

11. Skurnik, M.; Venho, R.; Bengoechea, J.A.; Moriyón, I. The lipopolysaccharide outer core of Yersinia enterocolitica serotype O:3 is required for virulence and plays a role in outer membrane integrity. Mol. Microbiol. 1999, 31, 1443-1462. [CrossRef] [PubMed]

12. Erridge, C.; Bennett-Guerrero, E.; Poxton, I.R. Structure and function of lipopolysaccharides. Microbes. Infect. 2002, 4, 837-851. [CrossRef]

13. Cuervo, A.; Fabrega-Ferrer, M.; Machon, C.; Conesa, J.J.; Fernandez, F.J.; Perez-Lique, R.; Perez-Ruiz, M.; Pous, J.; Vega, M.C.; Carrascosa, J.L.; et al. Structures of T7 bacteriophage portal and tail suggest a viral DNA retention and ejection mechanism. Nat. Commun. 2019, 10, 3746-3756. [CrossRef]

14. Molineux, I.J. No syringes please, ejection of phage T7 DNA from the virion is enzyme driven. Mol. Microbiol. 2001, 40, 1-8. [CrossRef] [PubMed]

15. Garcia-Doval, C.; van Raaij, M.J. Structure of the receptor-binding carboxy-terminal domain of bacteriophage T7 tail fibers. Prac. Natl. Acad. Sci. USA 2012, 109, 9390-9395. [CrossRef] [PubMed]

16. Veesler, D.; Cambillau, C. A common evolutionary origin for tailed-bacteriophage functional modules and bacterial machineries. Microbiol. Mol. Biol. Rev. 2011, 75, 423-433. [CrossRef]

17. Muhlenhoff, M.; Stummeyer, K.; Grove, M.; Sauerborn, M.; Gerardy-Schahn, R. Proteolytic processing and oligomerization of bacteriophage-derived endosialidases. J. Biol. Chem. 2003, 278, 12634-12644. [CrossRef] 
18. Schulz, E.C.; Dickmanns, A.; Urlaub, H.; Schmitt, A.; Muhlenhoff, M.; Stummeyer, K.; Schwarzer, D.; Gerardy-Schahn, R.; Ficner, R. Crystal structure of an intramolecular chaperone mediating triple-beta-helix folding. Nat. Struct. Mol. Biol. 2010, 17, 210-215. [CrossRef]

19. Steven, A.C.; Trus, B.L.; Maizel, J.V.; Unser, M.; Parry, D.A.D.; Wall, J.S.; Hainfeld, J.F.; Studier, F.W. Molecular substructure of a viral receptor-recognition protein. The gp17 tail-fiber of bacteriophage T7. J. Mol. Biol. 1988, 200, 351-365. [CrossRef]

20. Pyra, A.; Urbańska, N.; Filik, K.; Tyrlik, K.; Brzozowska, E. Biochemical features of the novel Tail Tubular Protein A of Yersinia phage phiYeO3-12. Sci. Rep. 2020, 10, 4196-4206. [CrossRef]

21. Eschenfeldt, W.H.; Stols, L.; Sanville Millard, C.; Joachimiak, A.; Donnelly, M.I. A family of LIC vectors for high-throughput cloning and purification of proteins. Methods Mol. Biol. 2009, 498, 105-115. [PubMed]

22. Laemmli, U.K. Cleavage of structural proteins during the assembly of the head of bacteriophage T4. Nature 1970, 227, 680-685. [PubMed]

23. Louis-Juene, C.; Andrade-Navarro, M.A.; Perez-Iratxeta, C. Prediction of protein secondary structure from circular dichroism using theoretically derived spectra. Proteins 2012, 80, 374-381.

24. Smith, P.K.; Krohn, R.I.; Hermanson, G.T.; Mallia, A.K.; Gartner, F.H.; Provenzano, M.D.; Fujimoto, E.K.; Goeke, N.M.; Olson, B.J.; Klenk, D.C. Measurement of protein using bicinchoninic acid. Anal. Biochem. 1985, 150, 76-85. [PubMed]

25. Hernandez, E.; Girardet, M.; Ramisse, F.; Vidal, D.; Cavallo, J.D. Antibiotic susceptibilities of 94 isolates of Yersinia pestis to 24 antimicrobial agents. J. Antimicrob. Chemother. 2003, 52, 1029-1031. [PubMed]

26. NanoTemper, Version 2018; PR.ThermControl \& PR.StabilityAnalysis Software: San Francisco, CA, USA, 2018.

27. Pyra, A.; Brzozowska, E.; Pawlik, K.; Gamian, A.; Dauter, M.; Dauter, Z. Tail tubular protein A: A dual-Function tail protein of Klebsiella pneumoniae bacteriophage KP32. Scient. Rep. 2017, 7, 2223. [CrossRef]

28. Brzozowska, E.; Pyra, A.; Pawlik, K.; Janik, M.; Górska, S.; Urbańska, N.; Drulis-Kawa, Z.; Gamian, A. Hydrolytic activity determination of Tail Tubular Protein A of Klebsiella pneumoniae bacteriophages towards saccharide substrates. Sci. Rep. 2017, 7. [CrossRef]

29. Altschul, S.F.; Madden, T.L.; Schaffer, A.A.; Zhang, J.; Zhang, Z.; Miller, W.; Lipman, D.J. Gapped BLAST and PSI-BLAST: A new generation of protein database search programs. Nucleic Acids Res. 1997, 25, 3389-3402.

30. Sievers, F.; Wilm, A.; Dineen, D.; Gibson, T.J.; Karplus, K.; Li, W.; Lopez, R.; McWilliam, H.; Remmert, M.; Söding, J.; et al. Fast, scalable generation of high-quality protein multiple sequence alignments using Clustal Omega. Mol. Syst. Biol. 2011, 7, 539.

31. Soding, J.; Biegerd, A.; Lupas, A.N. The HHpred interactive server for protein homology detection and structure prediction. Nucleic Acids Res. 2005, 33, 243-248.

32. Kemp, P.; Garcia, L.R.; Molineux, L.J. Changes in bacteriophage T7 virion structure at the initiation of infection. Virology 2005, 340, 307-317. [CrossRef] [PubMed]

33. Kelley, L.; Mezulis, S.; Yates, C.; Wass, M.; Sternberg, N. The Phyre2 web portal for protein modeling, prediction and analysis. Nat. Protoc. 2015, 10, 845-858. [CrossRef] [PubMed]

34. Zhang, Y. I-TASSER server for protein 3D structure prediction. BMC Bioinform. 2008, 9, 40. [CrossRef] [PubMed]

35. Waterhouse, A.; Bertoni, M.; Bienert, S.; Studer, G.; Tauriello, G.; Gumienny, R.; Heer, F.T.; de Beer, T.A.P.; Rempfer, C.; Bordoli, L.; et al. SWISS-MODEL: Homology modelling of protein structures and complexes. Nucleic. Acids Res. 2018, 46, W296-W303. [CrossRef] [PubMed]

36. Haggård-Ljungquist, E.; Halling, C.; Calendar, R. DNA sequences of the tail fiber genes of bacteriophage P2: Evidence for horizontal transfer of tail fiber genes among unrelated bacteriophages. J. Bacteriol. 1992, 174, 1462-1477. [CrossRef] [PubMed]

37. Garcia-Doval, C.; Castón, J.R.; Luque, D.; Granell, M.; Otero, J.M.; Llamas-Saiz, A.L.; Renouard, M.; Boulanger, P.; van Raaij, M.J. Structure of the receptor-binding carboxy-terminal domain of the bacteriophage T5 L-shaped tail fibre with and without its intra-molecular chaperone. Viruses 2015, 7, 6424-6440. [CrossRef]

38. Bharat, T.A.M.; Kureisaite-Ciziene, D.; Hardy, G.G.; Yu, E.W.; Devant, J.M.; Hagen, W.J.H.; Brun, Y.V.; Briggs, J.A.G.; Lowe, J. Structure of the hexagonal surface layer on Caulobacter crescentus cells. Nat. Microbiol. 2017, 2, 17059. [CrossRef] 
39. Szczesny, P.; Linke, D.; Ursinus, A.; Bar, K.; Schwarz, H.; Riess, T.M.; Kempf, V.A.J.; Lupas, A.N.; Martin, J.; Zeth, K. Structure of the head of the bartonella adhesin BadA. PLoS Pathog. 2008, 4, e1000119. [CrossRef]

40. Białas, N.; Kasperkiewicz, K.; Radziejewska-Lebrecht, J.; Skurnik, M. Bacterial cell surface structures in Yersinia enterocolitica. Arch. Immunol. Ther. Exp. 2012, 60, 199-209. [CrossRef]

Sample Availability: Samples of the compounds TTFBgp12, TFPgp17 are available from the authors.

(C) 2020 by the authors. Licensee MDPI, Basel, Switzerland. This article is an open access article distributed under the terms and conditions of the Creative Commons Attribution (CC BY) license (http://creativecommons.org/licenses/by/4.0/). 